\title{
Perspectives on making cartographic knowledge accessible to software developers
}

\author{
Amy L. Griffin,,${ }^{\mathrm{a}} *$ Serena Coetzee,${ }^{\mathrm{b}}$ Petr Kubicek, ${ }^{\mathrm{c}}$ Silvana P. Camboim, ${ }^{\mathrm{d}}$ Dalia E. Varanka, ${ }^{\mathrm{e}}$ \\ Francis Harvey, ${ }^{\mathrm{f}}$ Franz-Josef Behr, ${ }^{\mathrm{g}} \mathrm{Jan}$ Brus ${ }^{\mathrm{h}}$ \\ ${ }^{a}$ School of Science, RMIT University, Melbourne, Australia, amy.griffin@ rmit.edu.au \\ ${ }^{b}$ Department of Geography, Geoinformatics and Meteorology, University of Pretoria, Pretoria, South Africa, \\ serena.coetzee@up.ac.za \\ ${ }^{c}$ Department of Geography, Faculty of Science, Masaryk University, Brno, Czechia, kubicek@geogr.muni.cz. \\ ${ }^{d}$ Departamento de Geomática, Universidade Federal do Paraná,Brazil, silvanacamboim@ufpr.br \\ e U.S. Geological Survey, USA, dvaranka@usgs.gov \\ ${ }^{f}$ Leibniz Institute of Regional Geography, University of Leipzig, Germany, and University of Warsaw, Poland, \\ F_Harvey@leibniz-ifl.de \\ g Stuttgart University of Applied Sciences, Stuttgart, Germany, franz-josef.behr@hft-stuttgart.de \\ ${ }^{h}$ Department of Geoinformatics, Palacky University Olomouc, Czechia, jan.brus@upol.cz \\ * Corresponding author
}

Keywords: workflow, map design, semantics, standardization, cartography

\begin{abstract}
:
Contemporary cartographic practice uses a range of computing technologies to build print and electronic medium maps. Some allow the creation only of simple maps, through manipulation of a constrained set of design parameters (e.g., Mapline or Google's MyMaps). Others allow the manipulation of an expansive range of design elements (e.g., ArcGIS Pro, QGIS, Mapbox). Many software tools for making maps are not built by cartographers, but rather by software engineers, some of whom have limited cartographic training or mapmaking experience. Among the tools developers may use to help them design and implement mapmaking software are standards, such as those developed by groups like the Open Geospatial Consortium (OGC).
\end{abstract}

The OGC's Portrayal Domain Working Group (DWG) focuses on developing standards to support the visual representation of geospatial information (de la Beaujardiere, 2006; Sorenson, 2019). Its 2019 Charter advocates for a common portrayal framework but focuses more on interoperability of design choices that have been made (encoding of symbols and styles) and less on guidance or good practice of design choices (i.e., which symbol or style to use) (Sorenson, 2019). Cartographic associations (e.g., ICA) have had relatively little involvement with the development of these standards. Thus, a question relevant to our community is to what extent do existing OGC standards reflect good cartographic design practices? Bocher \& Ertz (2018) pointed to the low quality of maps using open standards (OGC included). They suggested a stronger focus on cartographic theory in the core OGC Symbology encoding standards and developed a proposed conceptual basis for defining geographical data symbology (Bocher \& Ertz, 2020).

Periodically, researchers have tried to capture cartographic knowledge to help non-experts make good maps (Forrest, 1993; Brus et al., 2010; Tsorlini et al., 2017) using varied approaches, including expert systems, cartographic workflows, and cartographic ontologies. Software engineers, however, are usually not attempting to design software to entirely replace mapmakers but rather to support the cartographic design process. Their task is to apply a systematic, disciplined, quantifiable approach to the development, operation, and maintenance of software (Bourque \& Fairly, 2014) for which they need an understanding of the fundamentals of computer science, namely the systematic study of algorithms (i.e., workflows) and data structures (i.e., ontologies) (Gibbs \& Tucker, 1986). We consider how we might connect software engineers' knowledge of algorithms and data structures to cartographic expertise via cartographic workflows or cartographic ontologies, respectively.

Software engineers are familiar with workflows and often think in such terms (Wing, 2014). A workflow is the series of activities necessary to complete a task (ISO 12967-1:2020) and can be distinguished from an expert system in that it is not able to learn from experience and is therefore more constrained in its operations and outcomes (Aniba et al., 2009). As an unstructured problem, the cartographic design process is contingent in nature; design decisions are influenced by a multiplicity of contextual factors (Griffin et al., 2017). This characteristic suggests potentially it might help to combine workflows with design patterns (Coetzee \& Rautenbach, 2017), when seeking to communicate cartographic knowledge to software engineers.

Software engineers are also familiar with schema representation of data and information. Ontology design patterns (ODP) are general templates that describe entities and relations for topical information schemas. Their development 
often begins as concept maps or a set of natural language propositions stating the core constraints to be represented by the schema. They guide the design of applied ontologies by suggesting a formal logic semantic structure among sets of objects and literal annotation (Fig. 1). Concurrently, Knowledge Representation and Reasoning expertise has developed to make concept models dynamic through executable logic. Such general schemas are refined by communities in application to take local practices and approaches into account (D'Ignazio \& Klein, 2019).

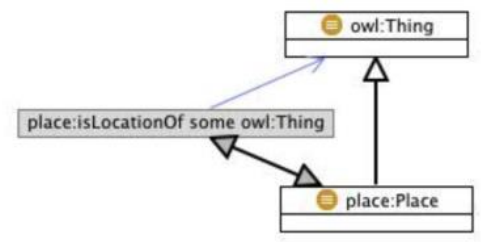

Figure 1. ODP example 'Place.' (Aldo Gangemi, Submissions:Place - Odp (ontologydesignpatterns.org))

As we are still at the beginning of this project, it is not yet clear to us whether workflows or ODPs will be a more fruitful approach to communicating cartographic design knowledge to software engineers. The topical subject domain content that will be formally specified in ODPs is normally debated among communities. Through this presentation, we are seeking community feedback on what cartographic workflows and map design core concepts are most important to make accessible to developers.

We present our initial thinking on what we consider to be core concepts and workflows. We believe our project can provide benefit to developers designing software for use by people and for the construction of automated tools. Cartographic workflows and ontologies can support the approach of building expert systems, or others based on artificial intelligence, to construct solutions that can help users build knowledge and helpful maps within the current panorama of an abundance of available spatial data.

\section{References:}

1. Aniba, M. R., Siguenza, S., Friedrich, A., Plewniak, F., Poch, O., Marchler-Bauer, A., \& Thompson, J. D. (2009). Knowledge-based expert systems and a proof-of-concept case study for multiple sequence alignment construction and analysis. Briefings in Bioinformatics, 10(1), 11-23.

2. Bocher, E., \& Ertz, O. (2020). OGC Symbology Conceptual Model: CorePart. http://www.opengis.net/doc/IS/SymCore/1.0. Last accessed 14 May 2021.

3. Bocher, E., \& Ertz, O. (2018). A redesign of OGC Symbology Encoding standard for sharing cartography. PeerJ Computer Science, 4: e143.

4. Bourque, P. \& Fairly, R. E. (eds.), (2014). Guide to the Software Engineering Body of Knowledge (SWEBOK) V3.0. IEEE Computer Society. https://www.computer.org/education/bodies-of-knowledge/softwareengineering/v3. Last accessed 17 May 2021.

5. Brus, J., Zdena, D., Kanok, J., \& Pechanec, V. (2010). Design of intelligent system in cartography. In 9th Roedunet IEEE International Conference (pp. 112-117). IEEE.

6. Coetzee, S., \& Rautenbach, V. (2017). A design pattern approach to cartography with big geospatial data. The Cartographic Journal, 54(4), 301-312.

7. De La Beaujardiere, J. (2006). OpenGIS Web Map Service (WMS) Implementation Specification. http://portal.opengeospatial.org/files/?artifact_id=14416. Last accessed 14 May 2021.

8. D’Ignazio, C. \& Klein, L. (2019). Data Feminism. Cambridge, MA, MIT Press.

9. Evans, E. (2003). Domain-driven design: Tackling complexity in the heart of software. Upper Saddle River, NJ, Addison-Wesley.

10. Forrest, D. (1993). Expert systems and cartographic design. The Cartographic Journal, 30(2), 143-148.

11. Gibbs N. E. and Tucker A. B. (1986). A model curriculum for a liberal arts degree in computer science. Communications of the ACM, 29(3), 202-210.

12. Griffin, A. L., White, T., Fish, C., Tomio, B., Huang, H., Sluter, C. R., Bravo, J. V. M., Fabrikant, S. I., Bleisch, S., Yamada, M. \& Picanço, P. (2017). Designing across map use contexts: A research agenda. International Journal of Cartography, 3(sup1), 90-114.

13. ISO. (2020). ISO 12967-1:2020, Health informatics - Service architecture (HISA) — Part 1: Enterprise viewpoint.

14. Sorenson, M. (2019). Portrayal Domain Working Group Charter. OGC Project Document 19-001r1. Open Geospatial Consortium. https://portal.ogc.org/files/?artifact_id=83869. Last accessed 14 May 2021.

15. Tsorlini, A., Sieber, R., Hurni, L., Klauser, H., \& Gloor, T. (2017). Designing a Rule-based Wizard for Visualizing Statistical Data on Thematic Maps. Cartographic Perspectives, 86, 5-23.

16. Wing J. M., (2014). Computational thinking benefits society. 40th Anniversary Blog on Social Issues in Computing. http://socialissues.cs.toronto.edu/index.html\%3Fp=279.html. Last accessed 17 May 2021. 